\title{
Influence of a nonlinear reference temperature profile on oscillatory Bénard-Marangoni convection
}

\author{
M. Dondlinger, ${ }^{1}$ P. Colinet, ${ }^{2, *}$ and P. C. Dauby ${ }^{1}$ \\ ${ }^{1}$ Université de Liège, Institut de Physique B5a, Allée du 6 Août 17, B-4000 Liège 1, Belgium \\ ${ }^{2}$ Université Libre de Bruxelles, Service de Chimie Physique E.P., 50, Avenue F. Roosevelt, Code Postal 165/62, \\ B-1050 Bruxelles, Belgium
}

(Received 27 March 2002; revised manuscript received 14 July 2003; published 31 December 2003)

\begin{abstract}
We analyze oscillatory instabilities in a fluid layer of infinite horizontal extent, heated from above or cooled from below, taking into account the nonlinearity of the reference temperature profile during the transient state of heat conduction. The linear stability analysis shows that a nonlinear reference temperature profile can have a strong effect on the system, either stabilizing or destabilizing, depending on the relative importance of buoyancy and surface tension forces. For the nonlinear analysis we use a Galerkin-Eckhaus method leading to a finite set of amplitude equations. In the two-dimensional (2D) case, we show the solution of these amplitude equations are standing waves.
\end{abstract}

DOI: 10.1103/PhysRevE.68.066310

PACS number(s): 47.20.Bp, 05.45.-a, 44.25.+f, 47.27.Te

\section{INTRODUCTION}

It is well known that, in a horizontal fluid layer heated from below, two mechanisms can be responsible for the onset of convection: the variation of density with temperature (buoyancy, or the Rayleigh-Bénard effect) and the variation of surface tension with temperature (thermocapillary, or the Marangoni-Bénard effect). In general, both effects combine, and give rise to the so-called Bénard-Marangoni instability [1-7].

In the case of a fluid layer heated from above, the situation drastically changes. For a long time, an unconditionally stable situation was predicted (both buoyancy and thermocapillary effects are stabilizing), but recent works ([8,9]), show that the conductive reference state may actually become unstable to oscillatory disturbances for sufficiently high temperature gradients and provided the buoyancy effect is sufficiently important with respect to the thermocapillary effect. Rednikov et al. [8] explained the appearance of such oscillatory instabilities by the linear interaction of internal and surface waves, which may lead to amplification. They calculated the marginal stability curves, corresponding to oscillatory perturbations when heating from above, showing that instabilities appear for very high values of the Marangoni number (of order $10^{6}$ ), such values being reachable for liquids of low viscosity (note that our Marangoni and Rayleigh numbers are proportional to the temperature difference between the top and the bottom of the fluid layer and are thus positive when the temperature at the top is higher than at the bottom as it is the case in this paper). They found that contrary to the situation when heating is from below, instability here requires the simultaneous action of both buoyancy and thermocapillary effects. For a given fluid, there is an optimal depth at which the system is most unstable. Furthermore, the system is shown to be always stable for shallow layers, for which surface tension dominates over buoyancy. In another paper, Bragard et al. $([10,9])$ found by

\footnotetext{
*Collaborateur Scientifique, Fonds National de la Recherche Scientifique.
}

an asymptotic analysis that the system may be unstable, even in the purely surface-tension-driven case (no buoyancy), provided one takes into account the nonlinearity of the reference temperature profile.

Another motivation of the present work is related to the recent experiments of Wierschem et al. [11]. In their setup, a fluid absorbs another overlying fluid with much lower density and surface tension and waves are observed at the very beginning of the equilibration process. It is important to note here that the equations governing mass and heat tranfers have the same form when effects specific to mass transfer such as accumulation and energy barriers are not taken into account in the interfacial boundary conditions. Thus the numerical results found in this paper on heat transfer are also applicable to mass transfer experiments ([12]). Wierschem et al. observed the appearance of waves at the beginning of the adsorption process, where the Marangoni number is clearly below the critical value for oscillatory instability as found by Rednikov et al. [8]. As these waves appear at the very beginning of the absorption process, one can think that the discrepancy between the experimental Marangoni number and the value predicted by Rednikov et al. can be due to the fact that the reference concentration (or temperature) profile is not yet linear when the instability takes place.

The purpose of this work is to perform a linear and a nonlinear study of oscillatory instabilities, and to analyze the influence of a nonlinear reference temperature profile on the behavior of the system. We first confirm the abovementioned asymptotic results by Bragard et al. ([10,9]). Furthermore, we show that, more generally, a nonlinear reference temperature profile can have either a stabilizing or a destabilizing effect on the system, depending on the ratio of buoyancy to surface-tension forces, on the way the temperature gradient is initially applied, on the time after the experiment is run, etc. Given that at the beginning of a Bénard experiment, the reference temperature profile is in general nonlinear, these results show that the effective critical Marangoni number can in some cases be much lower than the one calculated by using a linear reference profile. This means that the value of the temperature difference giving rise to the oscillatory instability can be much lower for a nonlinear ref- 


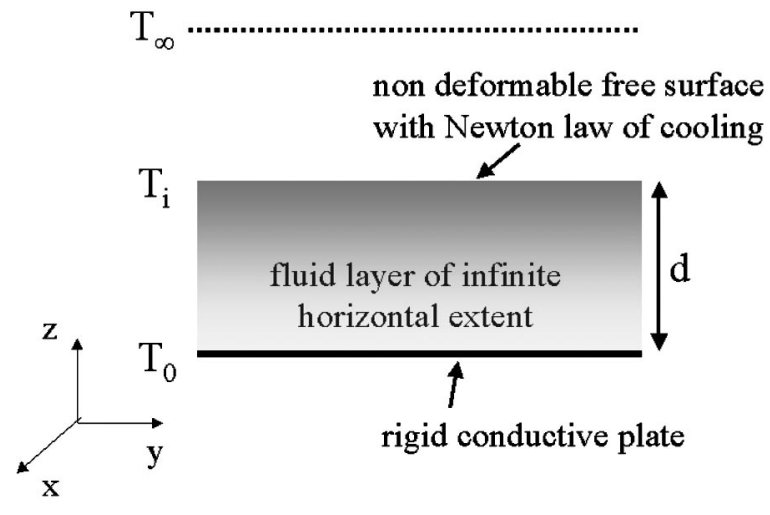

FIG. 1. System under study.

erence temperature profile than for a linear one.

In our approach, we assume that the temperature of the fluid layer is uniform for $t<t_{0}$. Then, at $t=t_{0}$, a temperature difference is imposed across the layer. The reference temperature profile we use for our stability analysis is then the time dependant solution of the heat equation in the fluid layer at rest, which is thus nonlinear at each time. Then a quasisteady stability analysis is carried out by assuming that the temperature profile can be "frozen" at each specified instant before analyzing the time evolution of infinitesimal perturbations with respect to the reference solution. This quasistatic approximation has already been used by many authors in the context of linear stability analyses (see, for example, [13-15]) and will also be justified a posteriori for our nonlinear study.

We consider both cases of heating from above and cooling from below. The quasisteady linear stability analysis is performed by using a normal mode technique, allowing the separation of variables, combined with a spectral TauChebyshev method. We also extend our analysis to the nonlinear regime, to determine the nature and the stability of the patterns formed above the linear stability threshold. Our approach is a generalized amplitude equations method adapted to the oscillatory problem using an iterative procedure that controls the smallness of the slaved amplitudes [16]. An analytical stability analysis of the typical wave patterns which appears in the 2D case is performed.

The paper is organized as follows. We introduce the physical system, define some dimensionless parameters and establish the basic equations in Sec. II. The linear problem is treated in Sec. III, yielding the instability threshold as a function of the nonlinearity of the reference temperature profile i.e., as a function of the "conductive" time after which the temperature difference is applied) and for different values of the ratio of buoyancy and surface tension effects. In Sec. IV we derive the nonlinear amplitude equations and we study the stability of the solutions found in the two dimensional case. Conclusions are drawn in Sec. V.

\section{PROBLEM FORMULATION}

The system under study is represented in Fig. 1. We consider a fluid layer of infinite horizontal extent and of thickness $d$. The system is confined between a lower rigid con-
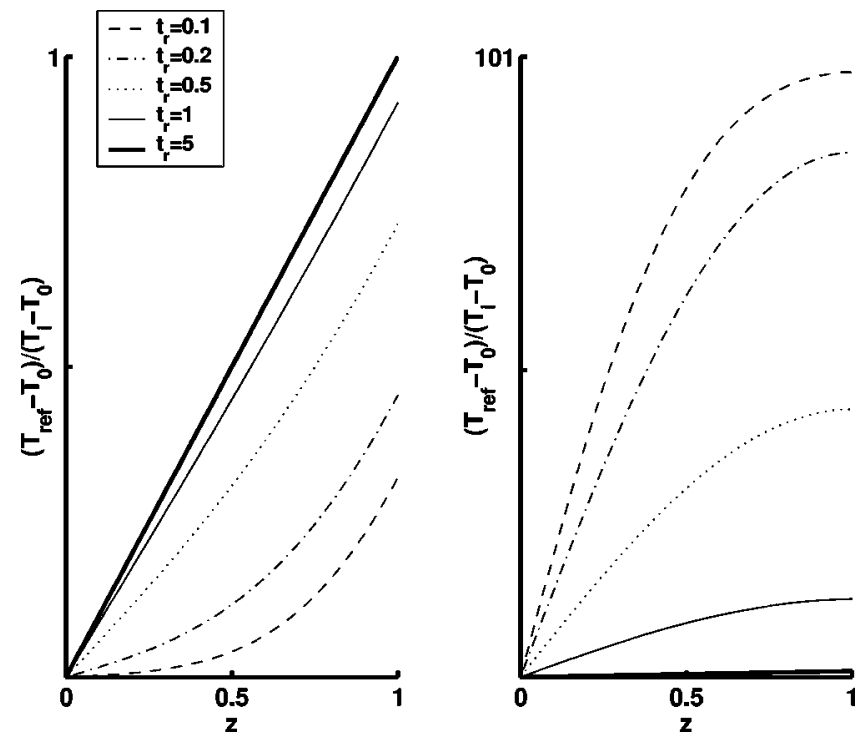

FIG. 2. Reference temperature profiles for different values of the dimensionless time $t_{r}$. Left figure: heating from above. Right figure: cooling from below $(\mathrm{Bi}=0.01)$.

ductive plate whose temperature $T_{0}$ is fixed and a flat upper free surface. To model heat transfers across this upper surface, we use the well-known Newton's law of cooling:

$$
-K \frac{d T}{d z}=h\left(T-T_{\infty}\right)
$$

where $K$ is the thermal conductivity of the liquid and $h$ is the heat transfer coefficient; $T$ is the temperature at the interface, $z$ is the vertical coordinate of a Cartesian reference frame with its origin at the bottom of the layer and its vertical axis pointing from the lower plate to the free surface and $T_{\infty}$ is the temperature far away.

As explained in the Introduction, a temperature difference is imposed at $t=t_{0}$ across the layer which was previously at a uniform temperature $T_{t<t_{0}}$. The reference temperature profile for the stability analysis is the time dependent solution of the heat equation. This profile can only be determined by a numerical approach and is represented in Fig. 2 for different values of the "conductive" time $t_{r}$. Both the heating from above $\left(T_{\infty}>T_{0}=T_{t<t_{0}}\right)$ and the cooling from below $\left(T_{t<t_{0}}\right.$ $=T_{\infty}>T_{0}$ ) cases are depicted. For sufficiently large values of $t_{r}$, both profiles become linear and the two situations are equivalent.

We present now the equations governing the perturbations with respect to this reference solution. First, note that we are working in the framework of the Boussinesq approximation and the mass density and surface tension are assumed to be linear functions of the temperature:

$$
\begin{gathered}
\rho=\rho_{0}-\alpha_{T}\left(T-T_{0}\right), \\
\sigma=\sigma_{0}-\gamma_{T}\left(T-T_{0}\right)
\end{gathered}
$$

where $\alpha_{T}$ and $\gamma_{T}$ are constant coefficients. 
In our stability analysis we consider that the (nonlinear) reference temperature profile is "frozen" at a given instant of time $t_{r}$; the reference temperature profile is unchanging during the application of the stability analysis. This quasistatic approximation is valid for the linear stability analysis since we are only concerned with whether or not the perturbations grow at any stage of the evolution of the reference temperature profile (see [13-15]). Concerning the nonlinear analysis we will discuss at the end of Sec. IV the domain where the quasistatic approximation is valid.

The variables are expressed in dimensionless form: lengths are scaled by the thickness $d$ of the layer, time by $d^{2} / \kappa$, with $\kappa$ the heat diffusivity of the liquid, velocity $\mathbf{v}$ $=(u, v, w)$ by $\kappa / d$ (vectors are written in bold characters), pressure $p$ by $\kappa \nu \rho_{0} / d^{2}$, with $\nu$ the kinematic viscosity of the liquid, and temperature $T$ by $\Delta T=\left(T_{i}-T_{0}\right)$, where $T_{i}$ is the temperature at the upper free surface in the reference state, calculated numerically at each time step $t_{r}$ of the evolution of the reference temperature profile.

The Prandtl, Rayleigh, Marangoni, and Biot nondimensional numbers are defined, respectively, by ( $g$ the acceleration due to gravity):

$$
\begin{gathered}
\operatorname{Pr}=\frac{\nu}{\kappa}, \\
\mathrm{Ra}=\frac{g \alpha_{T} d^{3} \Delta T}{\kappa \nu}, \\
\mathrm{Ma}=\frac{\gamma_{T} d \Delta T}{\kappa \nu \rho_{0}}, \\
\mathrm{Bi}=\frac{h d}{K} .
\end{gathered}
$$

In addition, as an alternative to the usual Marangoni and Rayleigh numbers, we have defined two different dimensionless numbers $\alpha$ and $\lambda$ by

$$
(1-\alpha) \frac{\mathrm{Ra}}{\mathrm{Ra}_{0}}=\alpha \frac{\mathrm{Ma}}{\mathrm{Ma}_{0}}
$$

and

$$
\lambda=\frac{\mathrm{Ra}}{\mathrm{Ra}_{0}}+\frac{\mathrm{Ma}}{\mathrm{Ma}_{0}}
$$

where $\mathrm{Ra}_{0}$ and $\mathrm{Ma}_{0}$ are two arbitrary constants which are, respectively, fixed at 669 and 79.607 in the following. The parameter $\alpha$ is a measure of the relative importance of the buoyancy effect with regard to the thermocapillary effect and can vary between zero and one ( $\alpha=0$ corresponds to pure thermocapillarity and $\alpha=1$ to pure buoyancy). This parameter is related to the well-known dynamic Bond number $\mathrm{Bo}_{d}=\mathrm{Ra} / \mathrm{Ma}$ through the following relation:

$$
\mathrm{Bo}_{d}=\frac{\mathrm{Ra}_{0} \alpha}{\mathrm{Ma}_{0}(1-\alpha)}
$$

Parameter $\lambda$ is directly proportional to the temperature gradient $(\lambda>0$ when the temperature at the top is higher than at the bottom). We now define the temperature, velocity, and pressure perturbations $\theta, \mathbf{v}^{\prime}$ and $\pi$ with respect to the conductive reference solution, as

$$
\begin{gathered}
T=T_{r e f}+\theta, \quad \mathbf{v}=\mathbf{v}_{r e f}+\mathbf{v}^{\prime}=\mathbf{v}^{\prime}=\left(u^{\prime}, v^{\prime}, w^{\prime}\right), \\
p=p_{r e f}+\pi .
\end{gathered}
$$

Under our assumptions, it is possible to neglect the term $\partial T_{\text {ref }} / \partial t$ in the heat equation, and the dimensionless equations governing the evolution of the perturbations (for convenience the primes have been dropped) are given by

$$
\begin{gathered}
\boldsymbol{\nabla} \cdot \mathbf{v}=0 \quad \text { (continuity) } \\
\partial_{t} \mathbf{v}+\mathbf{v} \cdot \boldsymbol{\nabla} \mathbf{v}=\operatorname{Pr}\left(-\boldsymbol{\nabla} \pi-\operatorname{Ra} \theta \mathbf{e}_{z}+\nabla^{2} \mathbf{v}\right) \quad(\text { momentum) } \\
\partial_{t} \theta+\mathbf{v} \cdot \boldsymbol{\nabla} \theta=-w \partial_{z} T_{r e f}+\nabla^{2} \theta \quad \text { (energy). }
\end{gathered}
$$

The boundary conditions (lower rigid conductive plate and nondeformable upper free surface with the Newton's law of cooling and Marangoni effect) are given by

$$
u=v=w=\theta=0
$$

at the bottom $z=0$, and

$$
\partial_{z} u-\operatorname{Ma} \partial_{x} \theta=\partial_{z} v-\operatorname{Ma} \partial_{y} \theta=\partial_{z} \theta+\operatorname{Bi} \theta=w=0
$$

at the top $z=1$.

\section{LINEAR STABILITY ANALYSIS}

To study the stability of the reference state we use a normal mode technique in horizontal directions, combined with a spectral Tau-Chebyshev method to solve the resulting equations for the vertical dependencies. First, Eqs. (11)(13) are linearized, the pressure field $\pi$ is eliminated by applying $\boldsymbol{\nabla} \times \boldsymbol{\nabla} \times$ to Eq. (12). Then, the temperature and velocity perturbations are written as the superposition of plane waves (normal modes) of the form

$$
(\mathbf{v}, \theta)=(\mathbf{V}(z), \Theta(z)) e^{i\left(k_{x} x+k_{y} y\right)} e^{\sigma t}
$$

where $\sigma$ is the complex growth rate of the perturbations and $k_{x}$ and $k_{y}$ are the components of the horizontal wave vector k. After standard calculations, we find the following equations:

$$
\begin{gathered}
\sigma\left(D^{2}-k^{2}\right) W-\operatorname{Pr}\left(D^{2}-k^{2}\right)^{2} W=\operatorname{Pr}_{\operatorname{Ra}_{0}} \alpha \lambda k^{2} \Theta \\
\sigma \Theta-\left(D^{2}-k^{2}\right) \Theta=-W D\left(T_{r e f}\right)
\end{gathered}
$$

where $D=\partial_{z}, W$ is the vertical component of $\mathbf{V}(z)$ and the boundary conditions are

$$
W=\Theta=D W=0
$$

at the bottom, and 

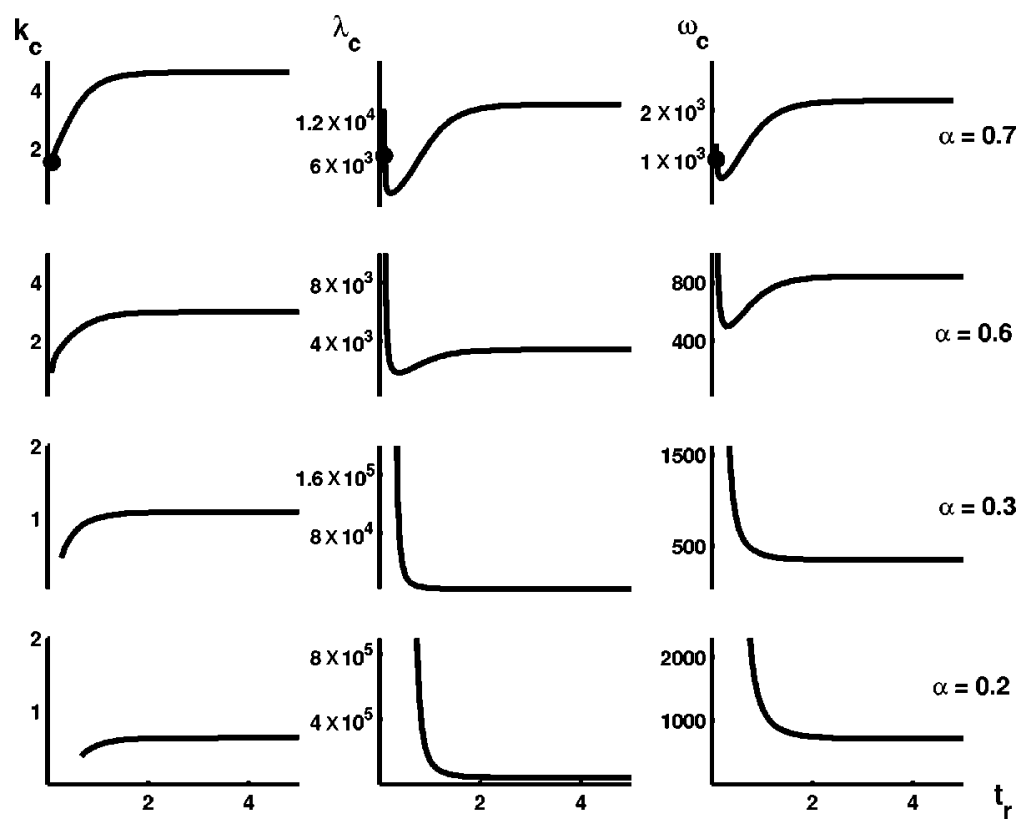

FIG. 3. $k_{c}$ (first column), $\lambda_{c}$ (second column), and $\omega_{c}$ (third column) as functions of time $t_{r}$ for different values of parameter $\alpha$ [first row: dominant buoyancy $(\alpha=0.7)$; last row: dominant thermocapillarity $(\alpha=0.2)]$ in the case of heating from above.

$$
W=D^{2} W-\operatorname{Ma}_{0}(1-\alpha) \lambda k^{2} \Theta=D \Theta+\operatorname{Bi} \Theta=0
$$

at the top.

We decompose $W(z)$ and $\Theta(z)$ in series of Chebyshev polynomials (spectral Tau-Chebyshev method). Then after projection of the equations on the Chebyshev polynomials and taking account of their orthogonality properties the final set of equations and boundary conditions can be written in the general form of an eigenvalue problem:

$$
A \mathbf{X}=\lambda B \mathbf{X}
$$

where $A$ and $B$ are two matrices depending on the parameters of the problem and $\mathbf{X}$ is the vector of the unknown coefficients of Chebyshev polynomials defining $W(z)$ and $\Theta(z)$. The eigenvalue $\lambda$ of the problem is a function $f$ of the parameters $\omega, \alpha, \operatorname{Pr}, \mathrm{Bi}, t_{r}$, and $k$, where $\omega=\operatorname{Im}(\sigma)$ is the oscillation pulsation of the perturbations $[\operatorname{Re}(\sigma)=0$ when seeking for marginal states]. As $\lambda$ is real, we have in fact two relations $\lambda=\operatorname{Re}\left[f\left(\omega, \alpha, \operatorname{Pr}, \mathrm{Bi}, t_{r}, k\right)\right]$ and $\operatorname{Im}\left[f\left(\omega, \alpha, \operatorname{Pr}, \mathrm{Bi}, t_{r}, k\right)\right]=0$, allowing us to find the pulsation $\omega$ and the corresponding value of $\lambda$ for given values of $\alpha$, $\mathrm{Pr}, \mathrm{Bi}, t_{r}$, and $k$. The critical $\lambda, \lambda_{c}$ is defined by

$$
\lambda_{c}=\min _{k} \lambda\left(\omega, \alpha, \operatorname{Pr}, \mathrm{Bi}, t_{r}, k\right)
$$

The wave number corresponding to $\lambda_{c}$ is the critical wave number $k_{c}$.

In Fig. $3, k_{c}, \lambda_{c}$, and $\omega_{c}$ are given as a function of time $t_{r}$ for different values of the parameter $\alpha$ (the other parameters are fixed: $\mathrm{Pr}=1, \mathrm{Bi}=0.01$ ) in the case of heating from above. Figure 4 concerns the case of cooling from below.
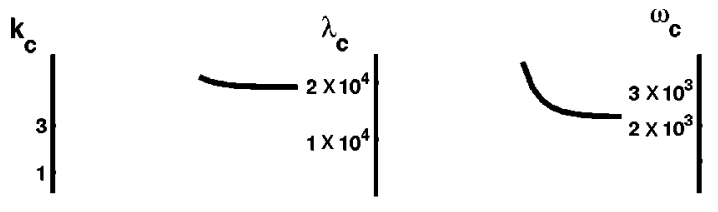

$\omega_{\mathbf{c}}$
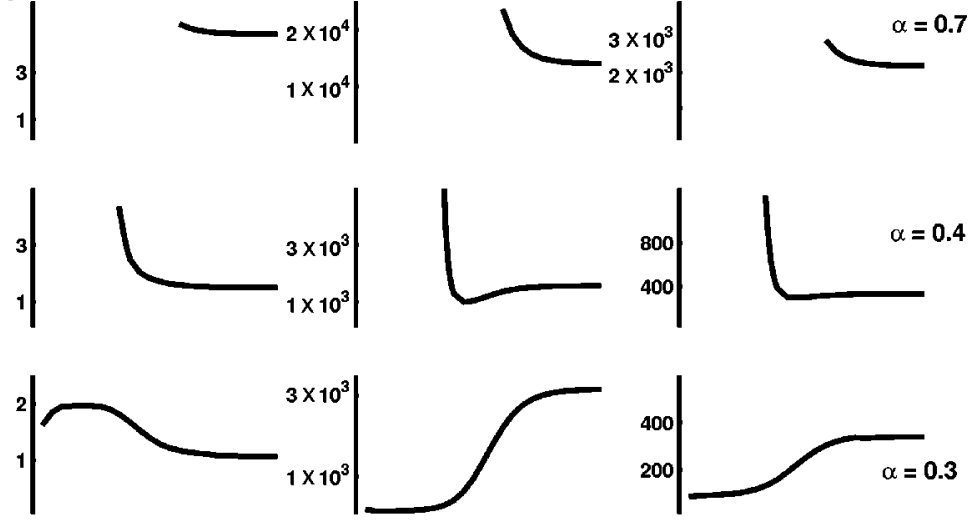

FIG. 4. $k_{c}$ (first column), $\lambda_{c}$ (second column), and $\omega_{c}$ (third column) as a function of time $t_{r}$ for different values of parameter $\alpha$ [first row: dominant buoyancy $(\alpha=0.7)$; last row: pure thermocapillarity $(\alpha=0)$ ] in the case of cooling from below.
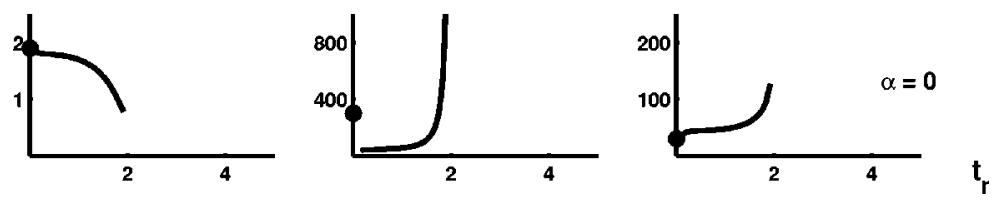
In the case of heating from above and for a low buoyancy effect $(\alpha \leqslant 0.1)$ the system is stable, both for a linear reference profile $\left(t_{r} \geqslant 4\right)$ and a nonlinear one (small $t_{r}$ ). For higher values of $\alpha$, oscillatory instabilities become possible as shown by Rednikov et al. [8] for a linear reference temperature profile. The case of a linear reference profile is indeed the most unstable one for not too strong buoyancy effect. But with still increasing influence of the buoyancy effect with respect to the surface tension effect $(\alpha \geqslant 0.5)$ we see that a nonlinear reference profile can have a destabilizing effect, i.e., the system is more unstable at the beginning of the heat/mass diffusion process. In this case the effective critical $\mathrm{Ma}$ (or $\mathrm{Ra}$, or $\lambda$ ) can be much lower than the one calculated using the assumption of a linear reference profile.

It is well known that in a purely surface-tension-driven situation $(\alpha=0)$ and with a linear reference temperature profile, oscillatory instabilities are not possible $([8,9])$. An asymptotic analysis by Bragard et al. ([10,9]) has shown that such oscillatory instabilities become possible in the case of cooling from below and at sufficiently small times $t_{r}$ (i.e., when the curvature $D^{2} T_{\text {ref }}$ of the temperature profile near the interface is sufficiently high). This result is confirmed by our analysis as one can see from Fig. 4.

We indeed see that in the case of cooling from below (Fig. $4)$, and without buoyancy $(\alpha=0)$, the system is stable for high values of $t_{r}$, but can be unstable for nonlinear reference temperature profiles. With the increasing importance of the buoyancy effect $(\alpha \geqslant 0.1)$, oscillatory instabilities appear even for linear reference profiles, but the nonlinear profiles are still the most unstable ones. Then for even higher values of $\alpha$ the case of a linear reference profile becomes the most unstable situation.

To summarize, we can stress that in both the heating from above or the cooling from below problem, the nonlinearity can have a destabilizing effect. In transient experiments such as those reported by Wierschem et al. ([11]) the instability could appear for a forcing condition on the system that is lower than the critical condition determined from the asymptotic reference solution.

Note also the evolution of the critical wave number $k_{c}$ and the critical pulsation $\omega_{c}$. The wave number increases with $\alpha$ in both cases of heating from above and cooling from below. The general tendency consists of an increase of the pulsation with $\alpha$, except that we observe a decrease of the pulsation between $\alpha=0.2$ and $\alpha=0.3$ for the case of heating from above. The wave number generally increases (decreases) with increasing $t_{r}$ in the case of heating from above (cooling from below).

Some numerical values of $\lambda_{c}, k_{c}$, and $\omega_{c}$ corresponding to Figs. 3 and 4 are given in Table I.

\section{NONLINEAR ANALYSIS}

The linear analysis allows us to determine the critical Marangoni (or Rayleigh, or $\lambda$ ) number, i.e., the critical temperature gradient above which the conductive state becomes unstable, the characteristic wave number of the flow pattern, and the corresponding pulsation. But the actual shape of the pattern can be obtained only via a nonlinear analysis. For this
TABLE I. Critical values corresponding to Figs. 3 and 4. [We selected the points where $\lambda_{c}$ is at its maximum value and those where the reference temperature is linear $\left(t_{r}=\infty\right)$.]

\begin{tabular}{lcccc}
\hline \hline$\alpha$ & $t_{r}$ & $k_{c}$ & $\lambda_{c}$ & $\omega_{c}$ \\
\hline \multicolumn{5}{c}{ Heating from above } \\
0.2 & $\infty$ & 0.6 & 38990 & 720 \\
0.3 & $\infty$ & 1.1 & 3170 & 347 \\
0.6 & 0.4 & 2.0 & 1830 & 513 \\
0.6 & $\infty$ & 3.0 & 3390 & 843 \\
0.7 & 0.24 & 2.3 & 1890 & 601 \\
0.7 & $\infty$ & 4.6 & 14040 & 2180 \\
& \multicolumn{5}{c}{} \\
\hline 0 & 0 & Cooling from below & \\
0.3 & 2.0 & 30 & 18 \\
0.3 & $\infty$ & 1.85 & 142 & 86 \\
0.4 & 1.1 & 3170 & 347 \\
0.4 & $\infty$ & 2.0 & 990 & 305 \\
0.7 & $\infty$ & 4.6 & 1570 & 332 \\
\hline \hline
\end{tabular}

purpose, we use a Galerkin-Eckhaus method, which consists of expanding the unknown perturbation fields in series of the eigenfunctions of the linear problem, then to introduce these expansions in the nonlinear equations and to project them onto the eigenfunctions of the adjoint linear problem. The infinite set of equations is then reduced to a finite number of ordinary differential equations by using a slaving principle adapted to the oscillatory problem (see [9]).

First we solve the linear equations (17)-(20) by fixing $\lambda$ $=\lambda_{c}$ and using the growth rate $\sigma$ of the perturbations as eigenvalue parameter. For each value of the wave number $k$ there exits an infinite set of eigenvalues $\sigma_{p}^{k}$, with $p$ being an integer running from one to infinity. The negative growth rates are ordered in such a way that $0 \geqslant \operatorname{Re}\left(\sigma_{p}^{k}\right)>\operatorname{Re}\left(\sigma_{q}^{k}\right)$ for $p<q$. The corresponding eigenfunctions have the form

$$
\left(\mathbf{v}_{p}^{\mathbf{k}}, \theta_{p}^{\mathbf{k}}\right)=\left(\mathbf{V}_{p}^{\mathbf{k}}(z), \Theta_{p}^{\mathbf{k}}(z)\right) e^{i\left(k_{x} x+k_{y} y\right)}
$$

The unknowns of the nonlinear problem (11)- (15)are then expanded as a series of these eigenfunctions,

$$
(\mathbf{v}, \theta)=\sum_{p=1}^{\infty} \sum_{\mathbf{k}} A_{p}^{\mathbf{k}}(t)\left(\mathbf{v}_{p}^{\mathbf{k}}, \theta_{p}^{\mathbf{k}}\right)
$$

where $A_{p}^{\mathbf{k}}$ are the time-dependent amplitudes and $\mathbf{k}$ can take all possible directions and moduli. After inserting (22) and (23) in the nonlinear equations (11)-(15), projection onto the eigenfunctions of the adjoint problem, integration by parts and noticing that the eigenfunctions are biorthogonal, one obtains the following general amplitude equations:

$$
\partial_{t} A_{p}^{\mathbf{k}}=\sigma_{p}^{\mathbf{k}} A_{p}^{\mathbf{k}}+\varepsilon \sum_{q=1}^{\infty} M_{q, p}^{\mathbf{k}} A_{q}^{\mathbf{k}}-\sum_{q, l=1}^{\infty} \sum_{\mathbf{k}_{1}, \mathbf{k}_{2}} N_{q, l, p}^{\mathbf{k}_{1}, \mathbf{k}_{2}, \mathbf{k}} A_{q}^{\mathbf{k}_{1}} A_{l}^{\mathbf{k}_{2}}
$$


where $\varepsilon=\left(\lambda-\lambda_{c}\right) / \lambda_{c}$ is the relative distance to the threshold and the matrices $M$ and $N$ are given by

$$
\begin{gathered}
M_{q, p}^{\mathbf{k}}=\frac{-\operatorname{Ra}_{0} \alpha \lambda_{c}\left\langle w_{p}^{\mathbf{k} *}, \theta_{q}^{\mathbf{k}}\right\rangle+\operatorname{Ma}_{0}(1-\alpha) \lambda_{c}\left\langle\partial_{z} w_{p}^{\mathbf{k} *}, \theta_{q}^{\mathbf{k}}\right\rangle_{z=1}}{\left\langle\operatorname{Pr}^{-1} v_{p}^{\mathbf{k} *} v_{p}^{\mathbf{k}}+\theta_{p}^{\mathbf{k} *} \theta_{p}^{\mathbf{k}}\right\rangle}, \\
N_{q, l, p}^{\mathbf{k}_{1}, \mathbf{k}_{2}, \mathbf{k}}=\frac{\operatorname{Pr}^{-1}\left\langle v_{p}^{\mathbf{k} *}, v_{q}^{\mathbf{k}_{1}} \cdot \nabla v_{l}^{\mathbf{k}_{2}}\right\rangle+\left\langle\theta_{p}^{\mathbf{k} *}, v_{q}^{\mathbf{k}_{1}} \cdot \nabla \theta_{l}^{\mathbf{k}_{2}}\right\rangle}{\left\langle\operatorname{Pr}^{-1} v_{p}^{\mathbf{k} *} v_{p}^{\mathbf{k}}+\theta_{p}^{\mathbf{k} *} \theta_{p}^{\mathbf{k}}\right\rangle},
\end{gathered}
$$

where the square brackets denote integration over the fluid volume and the asterisk denotes the eigenfunctions of the adjoint problem. This infinite number of ordinary differential equations for the amplitudes $A_{p}^{k}$ is then simplified by using a slaving method. The principle of the method consists in separating the set of eigenmodes in a first set $K_{c}$, containing the most unstable (critical) modes, and a second set $K_{s}$, containing the stable (slaved) modes. Since the real parts of the growth rates of the critical modes are close to zero, while the ones of the slaved modes have finite negative values, we can assume that near the threshold the moduli of the amplitudes belonging to the critical set are higher than the moduli of the amplitudes belonging to the slaved set. Under these circumstances we can rewrite Eq. (24) for the slaved modes, by restricting the summation to critical modes, which allows us to express the slaved amplitudes as functions of critical amplitudes only (as explained in [9]). We then obtain the equations for the critical modes by separating the sums on critical and slaved modes in the basic equations (24) and replacing the slaved amplitudes by their expressions as a factor of critical ones. Neglecting terms of order higher than 3 , we finally get

$$
\begin{aligned}
\partial_{t} A_{p}^{\mathbf{k}}= & \sigma_{p}^{\mathbf{k}} A_{p}^{\mathbf{k}}+\varepsilon \sum_{\substack{q \\
A_{q}^{\mathbf{k}} \in K_{c}}} M_{q, p}^{\mathbf{k}} A_{q}^{\mathbf{k}} \\
& -\sum_{\substack{q, l, \mathbf{k}_{1}, \mathbf{k}_{2} \\
A_{q}^{\mathbf{k}_{1}}, A_{l}^{\mathbf{k}_{2}} \in K_{c}}} N_{q, l, p}^{\mathbf{k}_{1}, \mathbf{k}_{2}, \mathbf{k}} A_{q}^{\mathbf{k}_{1}} A_{l}^{\mathbf{k}_{2}} \\
& -\sum_{\substack{q, m, n, \mathbf{k}_{1}, \mathbf{k}_{3}, \mathbf{k}_{4} \\
A_{q}, A_{m} \\
\mathbf{k}_{3}, \mathbf{k}_{4} \in A_{n} \in K_{c}}} Z_{q, m, n, p}^{\mathbf{k}_{1}, \mathbf{k}_{3}, \mathbf{k}_{4}, \mathbf{k}_{1}} A_{q}^{\mathbf{k}_{1}} A_{m}^{\mathbf{k}_{3}} A_{n}^{\mathbf{k}_{4}}
\end{aligned}
$$

where

$$
\begin{aligned}
Z_{q, m, n, p}^{\mathbf{k}_{1}, \mathbf{k}_{3}, \mathbf{k}_{4}, \mathbf{k}}= & \sum_{\substack{r, \mathbf{k}_{5} \\
A_{r}^{\mathbf{k}_{5} \in K_{c}}}}\left(N_{q, r, p}^{\mathbf{k}_{1}, \mathbf{k}_{5}, \mathbf{k}}+N_{r, q, p}^{\mathbf{k}_{5}, \mathbf{k}_{1}, \mathbf{k}}\right) N_{m, n, r}^{\mathbf{k}_{3}, \mathbf{k}_{4}, \mathbf{k}_{5}} \\
& \times \frac{1}{i \operatorname{Im}\left(\sigma_{m}^{\mathbf{k}_{3}}+\sigma_{n}^{\mathbf{k}_{4}}\right)-\sigma_{r}^{\mathbf{k}_{5}}} .
\end{aligned}
$$

In principle an infinite number of slaved modes should be considered to calculate the cubic coefficients in Eq. (25), but in practice the number of slaved modes which must be taken into account for each value of $k$ to ensure good convergence is limited to four or five.

Here for illustration we write the final expressions of the amplitude equations for the $2 \mathrm{D}$ case. We consider only two critical eigenmodes $A_{1}^{\mathbf{k}_{1}}$ and $A_{2}^{\mathbf{k}_{1}}$, where $\mathbf{k}_{1}=k_{c} \mathbf{e}_{x}$ is the critical eigenvector and $\sigma_{1}^{\mathbf{k}_{1}}=+i \omega_{c}$ and $\sigma_{2}^{\mathbf{k}_{1}}=-i \omega_{c}$ are the corresponding eigenvalues, $\omega_{c}$ being the critical pulsation. The coefficients of the amplitude equations are calculated at the critical point $\lambda_{c}$. The slaved wave vectors are $\mathbf{k}_{0}=\mathbf{k}_{1}-\mathbf{k}_{1}$ $=0$ and $\mathbf{k}_{2}=\mathbf{k}_{1}+\mathbf{k}_{1}=2 \mathbf{k}_{1}$. To simplify notation we note $A_{1}^{\mathbf{k}_{1}}=A$ and $A_{2}^{\mathbf{k}_{1}}=B$ and, from Eq. (25) we find the two complex amplitude equations:

$$
\begin{aligned}
& \partial_{t} A=c \varepsilon A-\gamma|A|^{2} A-\beta|B|^{2} A, \\
& \partial_{t} B=\bar{c} \varepsilon B-\bar{\gamma}|B|^{2} B-\bar{\beta}|A|^{2} B .
\end{aligned}
$$

This is a quite general expression, where only the coefficients $c, \gamma$, and $\beta$ depend on the particularities of the problem (see, for example, [10]). Constant amplitude solutions of these amplitude equations are the conductive state $(A=B$ $=0)$, standing waves $(|A|=|B| \neq 0)$ and traveling waves $(A$ $=0, B \neq 0$ or $A \neq 0, B=0)$. Traveling waves are stable if 0 $<\operatorname{Re}(c) \varepsilon$ and $0<\operatorname{Re}(\gamma)<\operatorname{Re}(\beta)$, while standing waves are stable if $0<\operatorname{Re}(c) \varepsilon, 0<\operatorname{Re}(\gamma)$ and $-\operatorname{Re}(\gamma)<\operatorname{Re}(\beta)$ $<\operatorname{Re}(\gamma)$ (see [2]).

In both cases (heating from above and cooling from below) and for any value of $\alpha$, we predict the appearance of standing waves above the linear stability threshold. Eventually, note that in all the cases investigated we recover the known results $([8,9])$ for a linear reference profile $\left(t_{r} \geqslant 4\right)$ : in this case the conductive solution is stable below some limiting value of $\alpha$ and standing waves appear above this limit, which is a check of our calculation.

In both cases (heating from above and cooling from below) and for any value of $\alpha$, we predict the appearance of standing waves above the linear stability threshold. Eventually, note that in all the cases investigated we recover the known results $([8,9])$ for a linear reference profile $\left(t_{r} \geqslant 4\right)$ : in this case the conductive solution is stable below some limiting value of $\alpha$ and standing waves appear above this limit, which is a confirmation of our calculation.

We close now this section with a detailed analysis of the validity of the quasistatic approximation in the context of our nonlinear approach. For this approximation to be acceptable, the dimensional time scale of the growth of the perturbations must be much smaller than the thermal diffusive time scale $\left(d^{2} / \kappa\right)$. Equations (27), (28) show that an estimate of the nondimensional time scale of the growth of the perturbations is given by $1 / \operatorname{Re}(c) \varepsilon$. This quantity must thus be much smaller than one for the quasistatic approximation to be valid, which means that $\varepsilon$ must be large enough. Otherwise stated, this means that the system must be sufficiently far from the threshold. But in that case, the amplitude method becomes questionable since its validity is, strictly speaking, limited to the close neighborhood of the linear stability limit. Even if it is well known that the qualitative results deduced 

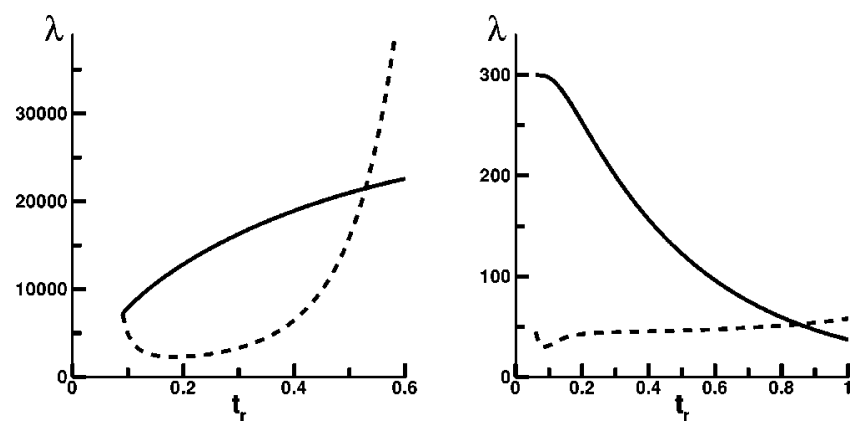

FIG. 5. The nondimensional temperature difference $\lambda$ between the top and the bottom of the layer as a function of $t_{r}$ is represented by the solid lines, while the dotted curves represent the functions of $t_{r}$ giving the value of $\lambda$ on the critical curve for a wave number always equal to $k_{c}$. Left: heating from above, $\alpha=0.7$ and $k_{c}$ $=1.53$. Right: cooling from below, $\alpha=0$ and $k_{c}=2$.

from amplitude equations are generally valid rather far from the threshold $([16,17])$ it is important to check in detail the consistency of our study.

This consistency will be examined for two typical cases, which have been selected because our results are significatively different from those corresponding to a linear reference temperature profile. First, let us analyze the case of heating from above with $\alpha=0.7$ and the situation for which the instability threshold is reached at $t_{r}=0.09, \lambda_{c}=7000$, $k_{c}=1.53$, and $\omega_{c}=975$ (see the dots in Fig. 3).

It is important to recall that the distance to the threshold varies with the conductive time $t_{r}$ since the reference temperature profile is not constant. In the case of heating from above, Fig. 2 shows that $T_{i}$ increases with time, which means that $\varepsilon$ also increases from zero at the threshold to positive values later on. Figure 5 allows us to study in detail the growth rate $1 / \operatorname{Re}(c) \varepsilon$ of the critical mode. The solid line is the nondimensional temperature difference between the top and the bottom of the layer as a function of $t_{r}$ while the dotted line represents the function of $t_{r}$ giving the value of $\lambda$ on the critical curve for a wave number always equal to the critical $k_{c}=1.53$. The relative distance between these two curves is thus the value of $\varepsilon$ for the unstable mode defined by the dots in Fig. 3. This mode is actually excited for $t_{r}$ larger than 0.09 and smaller than 0.52 . The average value of the distance to threshold between these two values of $t_{r}$ is 2.6. Since the calculation of the coefficient of the amplitude equations gives $\operatorname{Re}(c)=8.5$ in the present situation, the average time scale of the growth of the perturbation can be estimated to 0.045 , which is indeed much smaller than 1 . This means that in the present example, the quasistatic approximation is valid and the amplitude of the unstable mode has sufficient time to saturate before the reference temperature profile has notably changed.

The next delicate point is then the validity of our amplitude method which is, strictly speaking, limited to the close neighborhood of the linear stability limit while the mean value of $\varepsilon$ is equal to 2.6 in our problem. To examine this question, we use pure numerical 2D calculations with a horizontal Fourier decomposition and a finite difference method along the vertical coordinate. The results of these calcula-

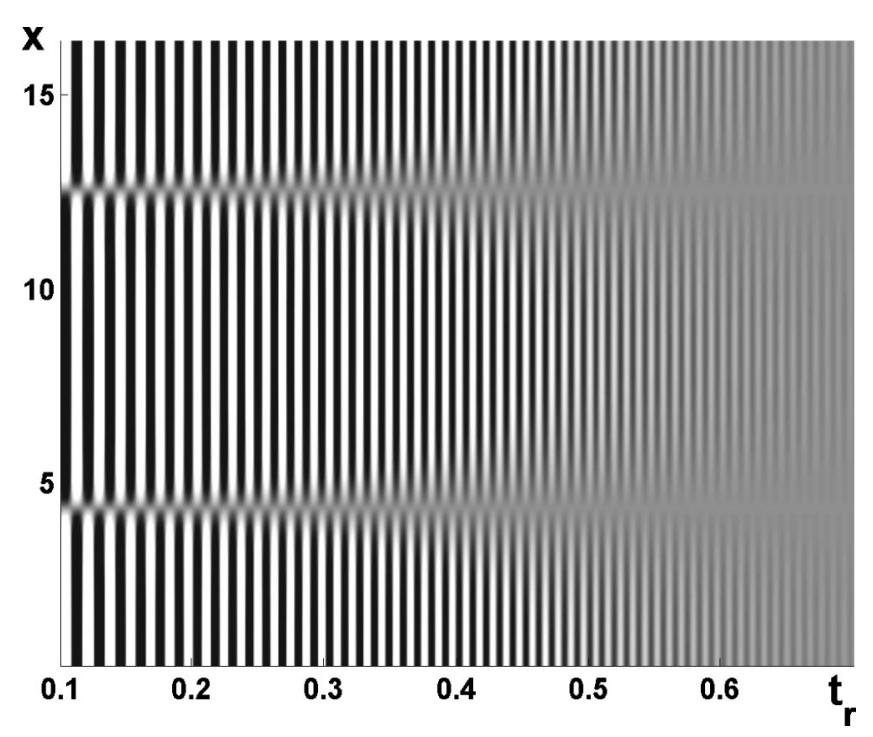

FIG. 6. Numerical standing waves in the case of heating from above $(\alpha=0.7, \lambda=7000)$. A gray-scale plot of the temperature field is presented. The values of $t_{r}$ on the horizontal axis correspond to the values in Fig. 5.

tions are given in Fig. 6 which clearly shows the appearance of a standing wave, as predicted by the amplitude method. A detailed analysis of this picture also enables us to determine the frequency of the wave. This frequency is seen to vary with time and we have checked that this numerical frequency is close to the frequency calculated along the solid line in Fig. 5. Eventually, one can also notice that, as expected, the standing wave progressively dies out when the two curves in Fig. 5 approach each other as time goes on.

As a second situation to check the validity of our quasistatic approach, we consider the case of cooling from below and a purely thermocapillary problem $(\alpha=0)$. If the reference temperature profile were assumed linear, it is well known that no instability is possible in this case. On the other hand, Fig. 4 shows that in the case of a nonlinear evolving temperature profile an instability is predicted for small values of $t_{r}$ while all perturbations should decay for large values of the time variable. The value of the nondimensional temperature difference at the beginning of the experiment is assumed to be 300; the most unstable wave number characterizing the critical mode is equal to 2 and the corresponding frequency is given by 18 . In this situation, Fig. 5 shows that the critical mode is indeed excited for values of $t_{r}$ smaller than 0.85 . With $\operatorname{Re}(c)=5.1$ and a mean value of $\varepsilon$ for $0<t_{r}<0.85$ equal to 2.7 , the mean time scale for the growth of the perturbation is equal to 0.072 , which justifies the application of the quasistatic analysis. Moreover, pure numerical calculations show that the standing waves predicted by the amplitude method actually appear for small $t_{r}$ and enventually die out for $t_{r} \geqslant \sim 0.85$.

In conclusion, we can assert the quasistatic approximation, leading to the amplitude equations and the prediction of standing waves above the threshold can be considered as a valid method to analyze the nonlinear evolution of our system. 


\section{CONCLUSIONS}

We have presented in this paper a linear and nonlinear stability analysis of a system formed by a laterally infinite horizontal fluid layer with a nondeformable surface, comparing the cases of heating from above and cooling from below and emphasizing the importance of the nonlinearity of the reference profile at the beginning of the experiment.

We showed in particular that when the layer is heated from above, a nonlinear reference profile has a destabilizing effect on the system, when buoyancy dominates over surface tension.

On the other hand, in the case of cooling from below, we found confirmation of the asymptotic results of Bragard et al., who found that oscillatory instabilities can be triggered even without buoyancy, provided the reference profile is sufficiently nonlinear. The nonlinear analysis of the patterns formed in the two-dimensional case shows that standing waves prevail over traveling waves in all cases investigated. Contrary to the case when considering a linear profile, we can find standing waves in the case of dominant surface tension effects, at the beginning of the heat (or mass) transfer experiment.
These results show that the assumption of a linear reference temperature profile can lead to wrong predictions, especially in the domain where surface tension effects are dominant when cooling is from below. Note finally that pure numerical calculations have also enabled us to prove the validity of the so-called quasistatic approximation that was used in the stability analysis.

\section{ACKNOWLEDGMENTS}

The authors want to thank Professor G. Lebon, T. Desaive, and S. Madruga (Liège University) as well as Professor J. Bragard (University of Navarra, Spain) for fruitful discussions. This research has been supported by the CIMEX and PRODEX-ESA projects under Contract No. 1429/00/ NL/SH and No. 14681/00/NL/S'Fe and by the European Union through Contract No. ICOPAC HRPN-CT-200000136. M.D. and P.C. acknowledge financial support, respectively, from the Fonds pour la Formation à la Recherche dans l'Industrie et dans l'Agriculture (Belgium) and from the Fonds National de la Recherche Scientifique (Belgium).
[1] E.L. Koschmieder, Bénard Cells and Taylor Vortices (Cambridge University Press, Cambridge, 1993).

[2] P. Bergé and M. Dubois, Contemp. Phys. 25, 535 (1984).

[3] P.G. Drazin and W. Reid, Hydrodynamic Stability (University Press, Cambridge, 1981).

[4] F.H. Busse, Rep. Prog. Phys. 41, 1929 (1978).

[5] C. Normand, Y. Pomeau, and M. Velarde, Rev. Mod. Phys. 49, 581 (1977).

[6] D.A. Nield, J. Fluid Mech. 19, 341 (1964).

[7] J.R.A. Pearson, J. Fluid Mech. 4, 489 (1958).

[8] A. Ye. Rednikov, P. Colinet, M.G. Velarde, and J.C. Legros, J. Fluid Mech. 405, 57 (2000).

[9] P. Colinet, J.C. Legros, and M.G. Velarde, Non Linear Dynamics of Surface Tension Driven Instabilities (Wiley-VCH, Berlin, 2001).
[10] J. Bragard, P. Colinet, A. Rednikov, and M.G. Velarde (private communication).

[11] A. Wierschem, M.G. Velarde, H. Linde, and W. Waldhelm, J. Colloid Interface Sci. 212, 365 (1999).

[12] Alexander A. Nepomnyashchy, Manuel G. Velarde, and Pierre Colinet, Interfacial Phenomena and Convection (Chapman and Hall/CRC, Boca Raton, FL, 2002).

[13] I.G. Currie, J. Fluid Mech. 29, 337 (1967).

[14] D.A. Nield, J. Fluid Mech. 71, 441 (1975).

[15] G. Lebon and A. Cloot, J. Non-Equilib. Thermodyn. 6, 15 (1981).

[16] P.C. Dauby, Th. Desaive, J. Bragard, and P. Cerisier, Phys. Rev. E 64, 066301 (2001).

[17] P.M. Parmentier, V.C. Regnier, G. Lebon, and J.C. Legros, Phys. Rev. E 54, 411 (1996). 